\title{
A method for establishing human primary gastric epithelial cell culture from fresh surgical gastric tissues
}

\author{
FAISAL AZIZ $^{1}$, XUESONG YANG ${ }^{1}$, QINGPING WEN ${ }^{2}$ and QIU YAN $^{1}$ \\ ${ }^{1}$ Department of Biochemistry and Molecular Biology, Dalian Medical University, Liaoning Provincial Core Lab \\ of Glycobiology and Glycoengineering, Dalian, Liaoning 116044; ${ }^{2}$ Department of Anesthesia, \\ The First Affiliated Hospital, Dalian Medical University, Dalian, Liaoning 116011, P.R. China
}

Received February 17, 2014; Accepted October 25, 2014

DOI: $10.3892 / \mathrm{mmr} .2015 .3692$

\begin{abstract}
At present, biopsy specimens, cancer cell lines and tissues obtained by gastric surgery are used in the study and analysis of gastric cancer, including the molecular mechanisms and proteomics. However, fibroblasts and other tissue components may interfere with these techniques. Therefore, the present study aimed to develop a procedure for the isolation of viable human gastric epithelial cells from gastric surgical tissues. A method was developed to culture human gastric epithelial cells using fresh, surgically excised tissues and was evaluated using immunocytochemistry, periodic acid-Schiff (PAS) staining and cell viability assays. Low cell growth was observed surrounding the gastric tissue on the seventh day of tissue explant culture. Cell growth subsequently increased, and at 12 days post-explant a high number of pure epithelial cells were detected. The gastric cancer cells exhibited rapid growth with a doubling time of 13-52 h, as compared to normal cells, which had a doubling time of 20-53 h. Immunocytochemical analyses of primary gastric cells revealed positive staining for cytokeratin 18 and 19, which indicated that the culture was comprised of pure epithelial cells and contained no fibroblasts. Furthermore, PAS staining demonstrated that the cultured gastric cells produced neutral mucin. Granulin and carbohydrate antigen 724 staining confirmed the purity of gastric cancer and normal cells in culture. This method of cell culture indicated that the gastric cells in primary culture consisted of
\end{abstract}

Correspondence to: Professor Qiu Yan, Department of Biochemistry and Molecular Biology, Dalian Medical University, Liaoning Provincial Core Lab of Glycobiology and Glycoengineering, Dalian, Liaoning 116044, P.R. China

E-mail: yanqiu63@126.com

Professor Qingping Wen, Department of Anesthesia, The First Affiliated Hospital, Dalian Medical University, Lvshun South Road, Dalian, Liaoning 116011, P.R. China

E-mail: wqp.89@163.com

Key words: human gastric cancer, Helicobacter pylori, gastric epithelial cell culture, stomach, cancer cell culture, normal cell culture mucin-secreting gastric epithelial cells, which may be useful for the study of gastric infection with Helicobacter pylori and gastric cancer.

\section{Introduction}

Helicobacter pylori (H. pylori) is a gram-negative, microaerophillic, flagellated bacteria, which affects $>50 \%$ of the world's population (1-3). Gastric cancer is a leading malignant disease in numerous countries, including China, Korea and Japan (4). H. pylori is ranked as a class I carcinogen by the International Agency for Research on Cancer (Lyon, France). $H$. pylori colonizes to the gastric mucosa and adheres to gastric epithelial cells; therefore, it is of interest to use human cells in the study of $H$. pylori infection (5). However, the availability of human gastric normal and cancer cell lines is limited and it is difficult to successfully culture gastric epithelial cells $(5,6)$.

Gastric biopsy tissues are widely used in gastric cancer research, but have various drawbacks (7). A significant limitation of using tissues from endoscopic biopsies is the lack of sufficient cell numbers for plating. For the successful growth and differentiation of epithelial cells a certain cell planting density and the use of the appropriate culture media is required (8). Gastric cell lines established from human gastric surgical tissues may prove more useful in the study of gastric infectious diseases (4). At present, gastric epithelial biology research relies on primary cultures generated from fresh surgical tissues (6). There are numerous advantages to this technique, including the maintenance of characteristics of the original tissue and low interference of stromal components. Surgical tissues are therefore suitable for use in the study of cell morphology, genetic characteristics, cell differentiation, invasion, metastasis and gastric cancer therapy (7).

Several methods have been reported for the isolation of cells from the gastric mucosa for cell culture (8). However, the successful isolation and subsequent culture of human gastric mucous epithelial cells has remained difficult due to numerous factors (8). The production of various gastric stromal factors, including fibroblasts, interferes with the analysis of gastric cancer cells. Furthermore, too great a number of passages of the cells altered the protein expression and mutation behavior of the cultured cells compared to those in vivo $(7,8)$. 
Therefore, the establishment of human gastric cell lines exhibiting a true epithelial phenotype is an important step in the study of gastric epithelial cells (9). The present study therefore aimed to develop a continuous human gastric epithelial cell culture from gastric surgical tissue, which may be used in the study of $H$. pylori gastric infections.

\section{Materials and methods}

Cell culture reagents and media. RPMI-1640, fetal bovine serum (FBS), trypsin/EDTA and penicillin/streptomycin were purchased from Invitrogen Life Technologies (Carlsbad, CA, USA). Polyclonal rabbit anti-H. pylori was purchased from Dako North America, Inc. (Carpinteria, CA, USA). Mouse anti-carbohydrate antigen 724 (CA724), amphotericin B, fibronectin and horseradish peroxidase (HRP)-conjugated anti-rabbit and anti-mouse immunoglobulin $\mathrm{G}$ were purchased from Santa Cruz Biotechnology Inc. (Dallas, TX, USA). Rabbit anti-proliferating cell nuclear antigen (PCNA), cytokeratin 18 and granulin (GRN) were purchased from ProteinTech Group, Inc. (Chicago, IL, USA). Cytokeratin 19 was purchased from ZSGB-BIO (Beijing, China). Tissue culture plates and flasks were purchased from Corning-Costar (Corning, New York, NY, USA). Hoechst 3358 was obtained from Beyotime Institute of Biotechnology (Haimen, China) and periodic acid was purchased from Sinopharm Group Co., Ltd (Beijing, China).

Preparation of fibronectin-coated flask. Fibronectin was dissolved in cell culture medium at a concentration of $100 \mu \mathrm{g} / \mathrm{ml}$. Subsequently, sufficient fibronectin solution $(3 \mathrm{ml})$ to provide a coating was added to a cell-culture flask and cell-culture plate and incubated at $37^{\circ} \mathrm{C}$ for $1-2 \mathrm{~h}$. The fibronectin solution was aspirated and rinsed with media, prior to storage at $4^{\circ} \mathrm{C}$.

Preparation ofmedia.RPMI-1640 media was supplemented with $20 \%$ FBS, $100 \mathrm{U} / \mathrm{ml}$ penicillin, $100 \mu \mathrm{g} / \mathrm{ml}$ streptomycin, $25 \mathrm{mM}$ hepes buffer (Tocris Bioscience, Bristol, UK), $20 \mathrm{mM}$ sodium bicarbonate (Tianjin KaiXin chemical industry, Tianjin, china) and $0.5 \mathrm{U} / \mathrm{ml}$ insulin (Sigma-Aldrich, St. Louis, MO, USA). The transport medium did not contain serum and was supplemented with $2 \mu \mathrm{g} / \mathrm{ml}$ amphotericin B (Sigma-Aldrich).

Human gastric surgical tissue procurement. Gastric tissues ( $\mathrm{n}=45$ ) were obtained from patients (age, 35-85) undergoing surgical gastrectomy. Gastric cancer tissues were obtained at the Dalian Medical First and Second Affiliated Hospitals (Dailan, China) between 2012 and 2013. Surgical tissues were removed from the tumor sections, adjacent tissue and normal gastric mucosa of 45 different patients. The specimens were collected in transport medium and transported to the research laboratory. All gastric tissue samples collected from the patients and the research protocols were performed in accordance with the Institutional Review Board of Dalian Medical University (Dailan, China).

Tissue processing and plating. Gastric tissues were placed in 100 -mm cell culture plates containing transport media and the fat, connective and necrotic sections of the tissue were removed. The remaining tissue was finely cut into $1-\mathrm{mm}^{3}$ sections. For the primary tumor culture, invasive areas were selected from the serosal surface, whenever possible, to decrease the chance of microbial contamination. Normal and cancerous tissue sections from each patient were arranged in fibronectin-coated culture flasks $(\mathrm{n}=45)$, with $0.5-\mathrm{cm}$ spacing between each tissue and incubated at $37^{\circ} \mathrm{C}$ with $5 \% \mathrm{CO}_{2}$. Initially, no media was added so that the tissue edges dried and adhered to the surface. Following three hours of incubation without media, when the edges of the tissue were sufficiently dry, the tissue was supplemented with sufficient trace medium $(1-2 \mathrm{ml})$ to cover the bottom of the flask. The media was replaced every $24 \mathrm{~h}$. Prior to replacing the media, the flask was stood vertically and following the change of media, the flask was carefully laid down in order to avoid the detachment of tissue pieces. Following six times of culture, tissues which had epidermal cells that were identifiable under a microscope were marked. Unmarked tissues were removed by curettage when replacing the culture medium. Any remaining fibroblasts were also scratched off, which resulted in a significant reduction in the number of fibroblast cells in the culture. Epithelial cells were confirmed by cytokeratin 18 and 19 antigen expression and gastric cancer cells were identified using GRN and CA724 markers. Cell proliferation was determined by immunocytochemical analysis of PCNA expression.

Gastric cell growth rate. A suspension of $1 \times 10^{5}$ cells was seeded in 35-mm plastic dishes in the culture medium. The number of cells was counted in triplicate at 24-h intervals for seven days using a hemocytometer (Sysmex, Kobe, Japan). The doubling times of the cell populations were estimated during the exponential growth phase.

Periodic acid-Schiff (PAS) staining. Cells were trypsinized and seeded onto cover slips. The sections were rinsed in phosphate-buffered saline (PBS), oxidized for $5 \mathrm{~min}$ in $0.5 \%$ periodic acid (Sinopharm Group Co., Ltd), rinsed once in PBS prior to the addition of Schiff's reagent (Sigma-Alrich) for $15 \mathrm{~min}$. The Schiff's reagent was subsequently removed and the sections were rinsed with tap water for $10 \mathrm{~min}$. Finally, the sections were counterstained with hematoxylin (Santa Cruz Biotechnology, Inc.) and cover-slips were mounted with mounting media for microscopic visualization (Olympus IX71; Olympus Corp., Tokyo, Japan).

Immunocytochemistry. Cells, which had been continuously passaged until they were pure epithelial cells, were grown on glass coverslips, fixed with $4 \%$ paraformaldehyde (Sigma-Aldrich) for $20 \mathrm{~min}$ and subsequently treated with $0.1 \%$ Triton-PBS (Merck, Darmstadt, Germany) for 10 min. Following being blocked with goat serum (Santa Cruz Biotechnology, Inc.) for $2 \mathrm{~h}$, cells were incubated with polyclonal rabbit anti-H. pylori (1:100), rabbit anti-cytokeratin 18 (1:100), rabbit anti-cytokeratin 19 (1:100), rabbit anti-PCNA (1:100), rabbit anti-GRN (1:100) or mouse anti-CA724 (1:100), at $4^{\circ} \mathrm{C}$ overnight. The next day, the sections were rinsed with PBS and subsequently incubated with their respective secondary antibody (HRP-conjugated anti-rabbit and anti-mouse IgG for $30 \mathrm{~min}$ at room temperature. Immunocytochemical staining was performed using an avidin-biotin peroxidase complex kit (ZSGB-BIO, Beijing, China). Samples were then mounted with mounting medium containing DAPI (Santa Cruz 

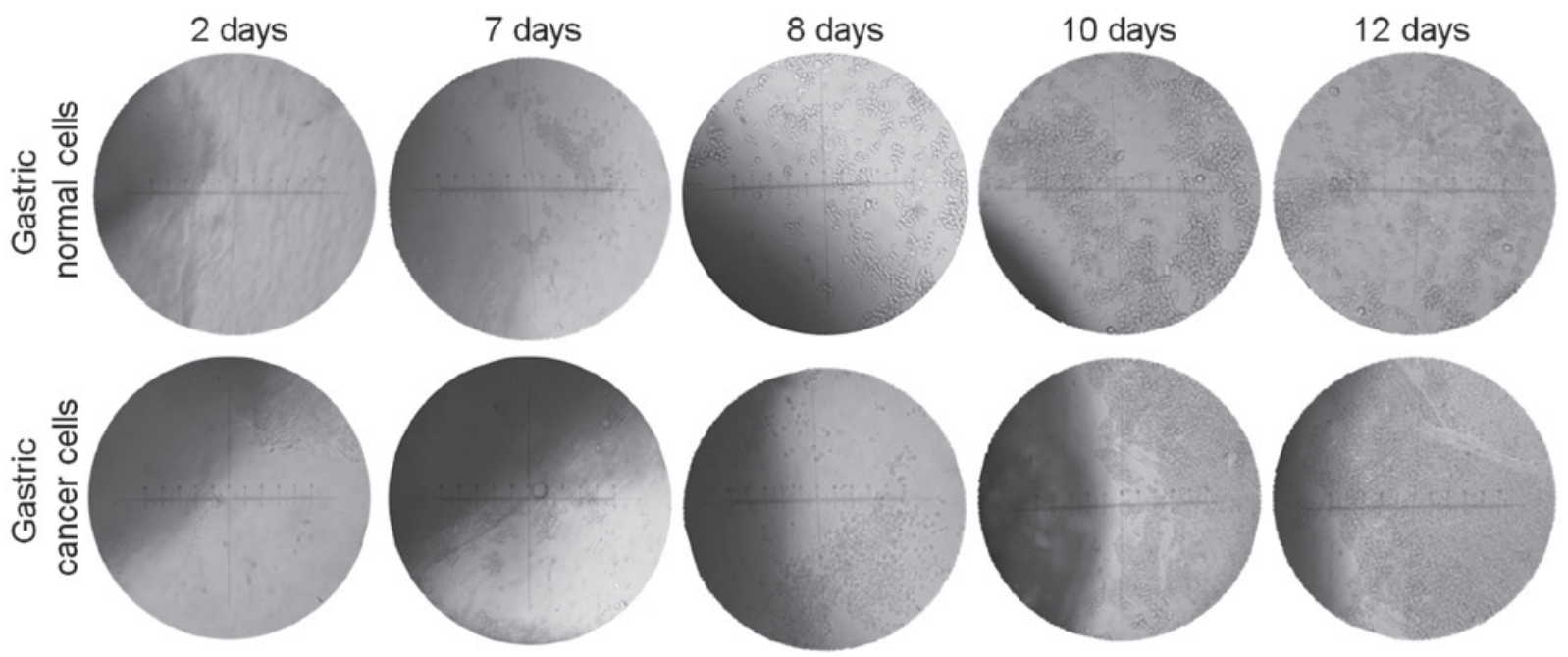

Figure 1. Epithelial status of human gastric epithelial cell cultures prepared from surgical specimens. Gastric explants demonstrated a gradual increase in the number of gastric epithelial cells from seven to 12 days of culture (magnification, x100).

Biotechnology, Inc. Images were captured with an inverted microscope (Olympus IX71; Olympus Corp., Tokyo, Japan).

Determination of mycoplasmic contamination using the Hoechst method. Cells were trypsinized, seeded on cover slips and fixed in $4 \%$ paraformaldehyde for $15 \mathrm{~min}$, prior to the addition of $100 \%$ cold methanol for $20 \mathrm{~min}$ at room temperature. Cells were subsequently rinsed three times with PBS and stained with Hoechst $(5 \mu \mathrm{g} / \mathrm{ml})$ for $15 \mathrm{~min}$ at room temperature. Finally, cells were rinsed with PBS and visualized under a fluorescence microscope (Olympus IX71; Olympus Corp.).

Statistical analysis. Statistical differences between test groups were analyzed using independent and paired Student's t-test and GraphPad Prism 5.03 software was used to statistical analyses (GraphPad Software, Inc., La Jolla, CA, USA). P<0.05 was considered to indicate as statistically significant difference between values.

\section{Results}

Gastric primary cell culture. Following seven days of culture of the explants of gastric tissue, epithelial cells began their outward migration of growth from the explants and started to increase their cell number with time. The cells reached high numbers at days 10-12 of culture (Fig. 1). Pure epithelial cells were cultured by the removal of fibroblast cells by curettage. The number of fibroblast cells in culture was significantly decreased by repeatedly scraping the tissue cultures until no fibroblast cells were detected. The passage number was dependent on the growth rate of tumor cells, which varied among patients. Cells were cultured for numerous months to maintain the cell morphology and growth rate. The cancer cells grew rapidly with an estimated doubling time of $13-52 \mathrm{~h}(\mathrm{P}<0.05)$, which was significantly increased compared with the doubling time of normal cells, which was 20-53 h $(\mathrm{P}<0.05$; Fig. 2 and Table I). In addition, the gastric cancer had a significantly higher cell count compared with the normal gastric cells at 2, 3 and 6 days $(\mathrm{P}<0.05)$. The cells were found to be free of contamination with mycoplasma (Fig. 3).

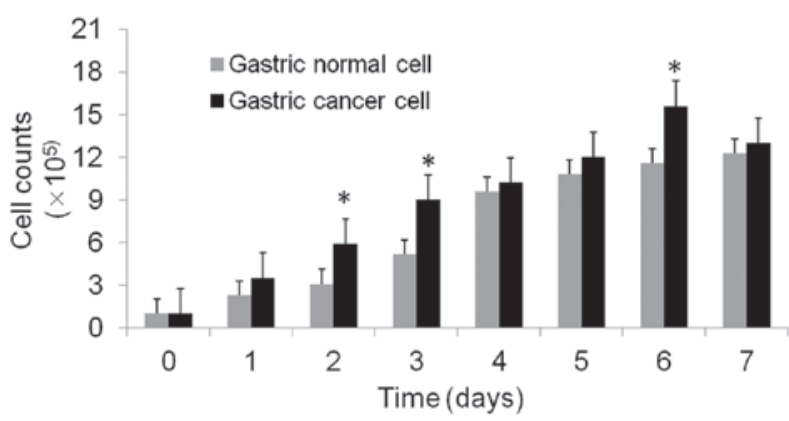

Figure 2. Bar diagram indicating the growth curve of the gastric culture cells. Normal gastric cells demonstrated low cell growth. Gastric cancer cells demonstrated higher cell growth at various time-points compared with the normal gastric cells $\left({ }^{*} \mathrm{P}<0.05\right)$. Values are presented as the mean. \pm standard error of the mean.
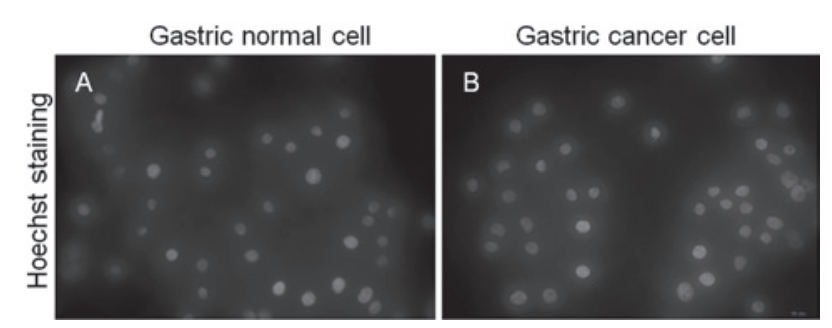

Figure 3. Gastric primary cultures were tested for mycoplasmic infection using the Hoechst 33258 method (magnification, x200). (A) Gastric normal and (B) gastric cancer cells were tested and found free of mycoplasmic contamination.

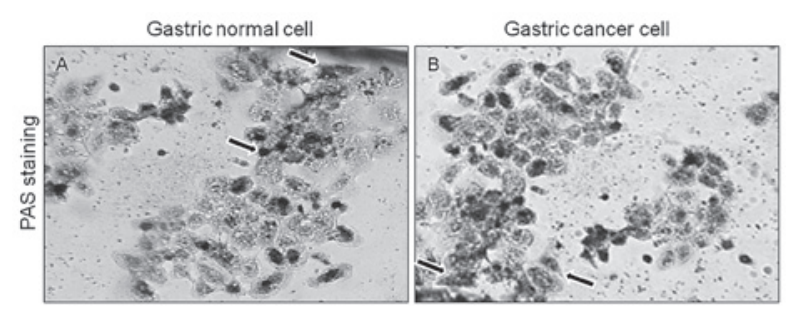

Figure 4. PAS staining with 30 min digestion. The arrows indicate purple cytoplasmic staining, which demonstrated the presence of neutral mucin within gastric epithelial cells (magnification, x200). PAS, periodic acid Schiff. 
Table I. Doubling time of gastric cancer and normal cells at various time-points.

\begin{tabular}{|c|c|c|c|c|c|}
\hline \multicolumn{3}{|c|}{ Normal cells } & \multicolumn{3}{|c|}{ Cancer cells } \\
\hline Cell count & Days $[\mathrm{n},(\mathrm{h})]$ & Doubling time (h) & Cell count & Days [n, (h)] & Doubling time (h) \\
\hline $1.0 \times 10^{5}$ & 0 & - & $1.0 \times 10^{5}$ & 0 & - \\
\hline $2.3 \times 10^{5}$ & $1(24)$ & 20 & $3.5 \times 10^{5}$ & $1(24)$ & 13 \\
\hline $3.1 \times 10^{5}$ & $2(48)$ & 29 & $5.9 \times 10^{5}$ & $2(48)$ & 19 \\
\hline $5.2 \times 10^{5}$ & $3(72)$ & 30 & $9.0 \times 10^{5}$ & $3(72)$ & 23 \\
\hline $9.6 \times 10^{5}$ & $4(120)$ & 37 & $10.2 \times 10^{5}$ & $4(120)$ & 36 \\
\hline $10.8 \times 10^{5}$ & $5(144)$ & 42 & $12.0 \times 10^{5}$ & $5(144)$ & 40 \\
\hline $11.6 \times 10^{5}$ & $6(168)$ & 48 & $15.6 \times 10^{5}$ & $6(168)$ & 42 \\
\hline $12.3 \times 10^{5}$ & $7(192)$ & 53 & $13.0 \times 10^{5}$ & $7(192)$ & 52 \\
\hline
\end{tabular}
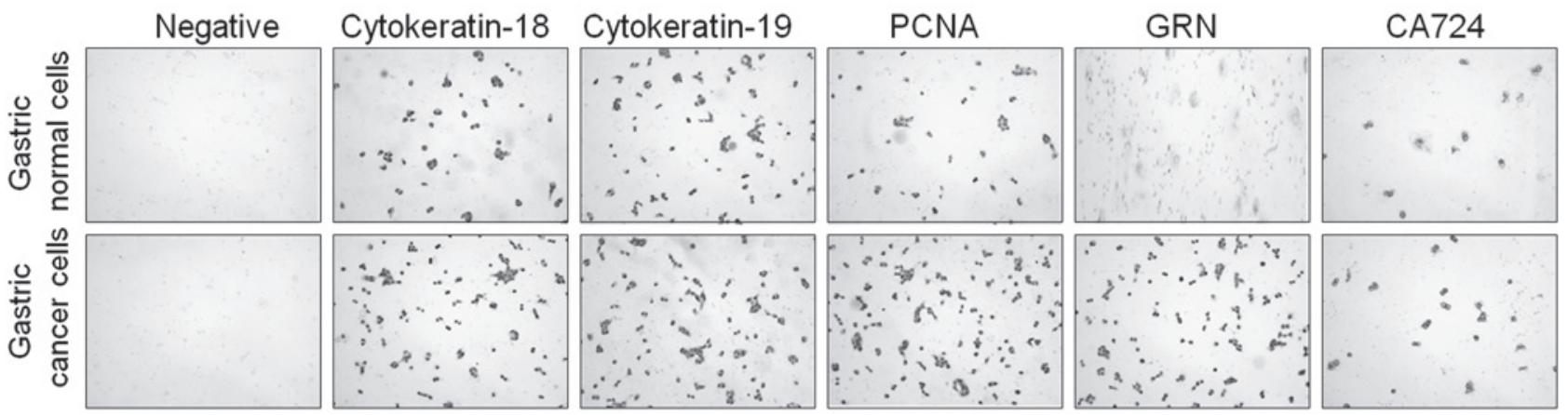

Figure 5. Staining of primary gastric cells with various markers in order to confirm epithelial and gastric cancer status. Positive staining for cytokeratin 18 and 19 was detected in the majority of the colony and confirmed that the gastric cancer and normal cells were pure epithelial cells. The highly positive staining for GRN and CA724 discriminated the gastric cancer cells from normal gastric cells (magnificantion, x100). Cell replications were determined using PCNA antibodies; one third of nuclei stained positive for PCNA, indicating active DNA synthesis and the potential for continued cell replication. Gastric cancer cells demonstrated a higher level of replication than normal cells. GRN, granulin; PCNA, proliferating cell nuclear antibody; CA724, carbohydrate antigen 724.

Expression of cytokeratin and neutral mucin demonstrates the gastric epithelial status of the primary culture cells. Primary gastric epithelial cells were stained with PAS reagent to determine the presence of neutral mucin and confirm the gastric epithelial origin of the cells. Gastric epithelial cells stained positive for neutral mucin by exhibiting a purple color (Fig. 4). The expression of antigens associated with gastric epithelial cells and gastric tumor markers was also evaluated. High expression levels of cytokeratin 18 and 19 were detected, which further confirmed the epithelial status of the gastric cancer and normal cells. To differentiate the gastric cancer from gastric normal cells, GRN and CA724 markers were used. The expression levels of GRN and CA724 were markedly higher in gastric cancer cells than those in normal cells. Furthermore, the high expression levels of PCNA in gastric cancer culture cells indicated evidence of high cell proliferation (Fig. 5).

\section{Discussion}

Gastric cancer is a multifactorial disease, caused by complex interactions between genetics, lifestyle and environmental factors $(10,11)$. There is a high prevalence of gastric cancer in China, which accounts for $42 \%$ of gastric cancer cases worldwide (12). Epidemiological studies conducted in Japan and China identified H. pylori infection as a significant risk factor for the development of gastric cancer (13). H. pylori infection is a major cause of gastritis and gastric ulcers, as well as gastric carcinoma (1). To date, studies have used gastric primary cell cultures to analyze $H$. pylori gastric infection, which colonizes to the gastric mucosa and adheres to gastric epithelial cells. It is difficult to establish gastric cell line cultures which exhibit a pure epithelial phenotype due to gastric stromal factor interference in the epithelial growth rate. A method for generating a gastric primary cell culture with high growth of gastric epithelial cells was therefore required. In the present study, fresh gastric surgical tissue was used to establish an effective method for culturing gastric epithelial from surgical gastric tissues $(5,7)$.

The selection of tissue, and specifically the gastric section of the tissue, is important for the successful development of a primary culture. Fresh gastric surgical tissues from patients $<60$ years of age have lower chances of bacterial contamination than those of patients aged $\geq 60$ years, which may have hypochlorhydria (5). In the present study, high growth of gastric epithelial cells with low contamination was found in specimens from younger patients (5). Furthermore, in gastric sections of the tissue, the tumor edges or junctions between the tumor and normal tissues were selected due to their previously reported high metabolic activity and ease of adherence (7). Ruttenl et al (8) reported an increased rate of growth of gastric epithelial cells 
from surgical specimens, compared with that of other specimens. Surgical tissues have numerous advantages, including the ease of removal from smooth muscle and that they are relatively simple to process (8). In order to avoid contamination, amphotericin B was added to the transport media to prevent fungal growth. Gastric primary cells were also tested for mycoplasmic contamination using Hoechst 33258, and no contamination was detected. Therefore, fresh gastric surgical tissue from patients of lower age was recommended to produce cultures of high growth rate and with low risk of contamination. Cell adherence to the flask surface is another important factor for the establishment of a successful primary culture (8). Fibronectin increases the rate of attachment and growth of the gastric epithelial cells. A previous study reported that fibronectin was a suitable substrate for mediating cell attachment (8).

During primary gastric culture, there are frequently problems with gastric stromal tissue components, including fibroblasts, which have potential cytotoxic effects and perturb the growth of pure gastric epithelial cells (14). In the present study, when cells surrounding the tissue began to appear following seven days of culture, no fibroblast cells were observed as the tissue was cultured in starting media, which contained no serum. Fibroblast growth depends upon the presence of serum; however, this is not a requirement for the growth of gastric cancer cells (14). For this reason, only gastric epithelial cell growth rather than fibroblast growth was observed in the cultured tissues. At a later stage, conventional media was used to promote rapid cell growth, and concurrent slow growth of fibroblast cells was detected (7). When the epithelial cell growth was high and had increased the number of epithelial cells, the media containing 20\% FBS was changed and fibroblast cells were removed by mechanical scraping. This method of primary culture was advantageous as it significantly inhibited the growth of fibroblast cells and a culture comprising $100 \%$ pure gastric epithelial cells was generated $(7,14)$. The culture medium may modulate the biological behavior of cultured cells (15). The appropriate use of media is an important factor for the successful growth and differentiation of gastric epithelial cells (8). It is also recognized that the majority of cells in vivo secrete endogenous growth factors to stimulate their own proliferation (8). Therefore, similar conditions may be achieved by using the appropriate media conditions during cell culture. In the present study, RPMI-1640 media, which is characterized by a combination of richness in trace elements, amino acids and high nutrient concentration, was used. Sodium bicarbonate and hepes buffer were also used for their abilities to maintain the $\mathrm{pH}$ of the media (15).

The characterization and investigation of cytokeratin expression by gastric cells is a simple method of determining epithelial nature (5). Cytokeratin markers were therefore used in the present study, to confirm that the primary gastric cells in culture were free from fibroblasts and comprised a pure epithelial gastric cell population. High levels of staining for cytokeratin 18 and 19 were identified in gastric normal and cancer primary cells, which confirmed the epithelial nature of the gastric culture cells. The cells that did not stain with cytokeratin 18 or 19 were presumed to be fibroblast cells (5). Mucin, which is found within the cells, may also be used to characterize gastric primary cultures (9). The PAS staining method was used to determine mucin expression within the gastric cells. In the present study, purple cytoplasmic staining was detected, which indicated the presence of neutral mucin within the gastric epithelial cells. This combination of neutral mucin and cytokeratin 18 and 19 expression demonstrated that the primary cultures were comprised of mucin-secreting gastric epithelial cells (5).

In order to differentiate gastric cancer cells from gastric normal cells, the expression of CA724 and GRN, which are associated with gastric tumor cells, was evaluated. High levels of CA724 and GRN staining are detected in gastric cancer cells, compared to those in normal gastric cells (16). CA724 is a specific gastric cancer marker used for the diagnosis of gastric diseases (17). Chen et al (18) demonstrated that CA724 was the most correlative and specific tumor biomarker for gastric cancer in the Chinese population. GRN is also a gastric cancer marker, which is highly expressed in gastric cancer and promotes cell proliferation, migration and invasion (19). Determination of the gastric cell proliferation rate aids the elucidation of the replicative ability of the primary cell culture. In order to determine the gastric culture cell proliferation rate, immunocytochemical staining was performed using PCNA antibodies. The results showed that difference in replication rate between gastric cancer and normal cells lies in the S-phase progression of the cell cycle. The replication rate of gastric cancer cells was higher than that of normal gastric cells. These results indicated that primary gastric cells had an active DNA synthesis and possessed the potential to continue gastric epithelial cell replication. Cell growth was examined using a trypan blue exclusion assay. Gastric cancer cell growth was markedly higher (13-52 h) than that of normal gastric cells (20-53 h). This result indicated that gastric cancer cells grow more rapidly than normal gastric cells (5).

In conclusion, the present study provided a method for the primary culture of gastric epithelial cells from fresh gastric surgical tissue. The advantage of the gastric primary culture method outlined is that the human tissue remained in medium and kept its activity intact with sufficient nutrition and adherence to the flask, which provided a suitable environment for continuous cell growth. The growth rate of gastric epithelial cells using this protocol was high and cultures were free from fibroblast cells. These cultured gastric epithelial cells may therefore be used to investigate the effects of $H$. pylori attachment to gastric epithelial cells and the therapeutic potential of various drugs against $H$. pylori infection.

\section{Acknowledgements}

The present study was supported by the China 973 grant (no. 2012CB822100) and the National Natural Science Foundation of China Research grant (nos. 30672753 and 31270866).

\section{References}

1. Aziz F, Sherwani SK, Akhtar SS and Kazmi SU: Development of an in-house enzyme-linked immunosorbent assay based on surface whole cell antigen for diagnosis of Helicobacter pylori infection in patients with gastroduodenal ulcer disease. World $\mathrm{J}$ Microbiol Biotechnol 30: 305-315, 2014.

2. Suerbaum S and Michetti P: Helicobacter pylori infection. N Engl J Med 347: 1175-1186, 2002

3. Torres J, Leal-Herrera Y,Perez-Perez G, et al: A community-based seroepidemiologic study of Helicobacter pylori infection in Mexico. J Infect Dis 178: 1089-1094, 1998. 
4. Park JG, Frucht H, LaRocca RV, et al: Characteristics of cell lines established from human gastric carcinoma. Cancer Res 50: 2773-2780, 1990.

5. Smoot DT, Sewchand J, Young K, et al: A method for establishing primary cultures of human gastric epithelial cells. Methods Cell Sci 22: 133-136, 2000

6. Chailler P and Ménard D: A new approach to primary culture of human gastric epithelium. Methods Mol Med 107: 217-236, 2005.

7. Liu G, Chai Y, Zhu X and Zhang Q: Explants culture of gastric tissue continuously in a small amount of medium. Cancer Res Prev Treat 2: 147-148, 2008 (In Chinese).

8. Rutten MJ, Campbell DR, Luttropp CA, et al: A method for the isolation of human gastric mucous epithelial cells for primary cell culture: A comparison of biopsy vs surgical tissue. Methods Cell Sci 18: 269-281, 1996.

9. Chailler P and Ménard D: Establishment of human gastric epithelial (HGE) cell lines exhibiting barrier function, progenitor, and prezymogenic characteristics. J Cell Physiol 202: 263-274, 2005.

10. Luk GD: Tumors of the stomach. In: Sleisenger and Fordtran's Gastrointestinal and Liver Disease: Pathophysiology/Diagnosis/ Management. Feldman M, Sleisenger MH and Scharschmidt B (eds). Vol 1. 6th edition. Saunders Co, Philadelphia, pp733-757, 1998.

11. Peek RM and Blaser MJ: Helicobacter pylori and gastrointestinal tract adenocarcinomas. Nat Rev Cancer 2: 28-37, 2002.
12. Parkin DM, Bray FI and Devesa SS: Cancer burden in the year 2000. The global picture. Eur J Cancer 37 (Suppl 8): S4-S66, 2001.

13. Fock KM, Talley NJ, Fass R, et al: Asia-Pacific consensus on the management of gastroesophageal reflux disease: update. J Gastroenterol Hepatol 23: 8-22, 2008.

14. Chew CS, Ljungström M, Smolka A and Brown MR: Primary culture of secretagogue-responsive parietal cells from rabbit gastric mucosa. Am J Physiol 256 (1 Pt 1): G254-G263, 1989.

15. Wu X, Lin M, Li Y, Zhao X and Yan F: Effects of DMEM and RPMI 1640 on the biological behavior of dog periosteum-derived cells. Cytotechnology 59: 103-111, 2009.

16. Isaka K, Nishi H, Nakada T, et al: Establishment and characterization of a new human cell line (EJ) derived from endometrial carcinoma. Hum Cell 15: 200-206, 2002.

17. Başoğlu M, Kiziltunç A, Akçay F, et al: Increased serum CA 72-4 levels in patients with gastrointestinal carcinoma. Turk J Med Sci 28: 259-263, 1998.

18. Chen XZ, Zhang WK, Yang K, Wang LL, et al: Correlation between serum CA724 and gastric cancer: multiple analyses based on Chinese population. Mol Biol Rep 39: 9031-9039, 2012.

19. Loei H, Tan HT, Lim TK, et al: Mining the gastric cancer secretome: identification of GRN as a potential diagnostic marker for early gastric cancer. J Proteome Res 11: 1759-1772, 2012. 